\title{
SKEW MONOIDAL CATEGORIES AND SKEW MULTICATEGORIES
}

\author{
JOHN BOURKE, STEPHEN LACK
}

\begin{abstract}
We describe a perfect correspondence between skew monoidal categories and certain generalised multicategories, called skew multicategories, that arise in nature.
\end{abstract}

\section{INTRODUCTION}

In linear algebra one meets the concept of a bilinear map $A \times B \rightarrow D$, and later learns that these are in bijection with linear maps $A \otimes B \rightarrow D$, so that one might say that bilinear maps are "classified" by the tensor product $A \otimes B$. Similarly there are trilinear maps $A \times B \times C \rightarrow D$ and these are classified by the tensor product $A \otimes B \otimes C$, which can be constructed out of the binary construction as either of the (isomorphic) objects $(A \otimes B) \otimes C$ and $A \otimes(B \otimes C)$. The most important properties of the tensor product, including coherence, all follow from the universal property, without the need for an explicit construction of the tensor product.

In many other examples of monoidal structures which are not given by a categorical (cartesian) product, it is likewise the case that maps out of a tensor product $A \otimes B$ can alternatively be described in terms of some more primitive notion, corresponding to bilinearity.

This idea of multilinear morphisms can be abstracted in the notion of multicategory [20], and in such a multicategory one can then ask whether or not there is a suitable corresponding tensor product, in which case the multicategory is said to be representable. In this case the corresponding tensor product forms part of a monoidal structure, and indeed the notion of a representable multicategory is equivalent to that of a monoidal category.

There are various intermediate structures between monoidal categories and multicategories, including the notion of colax monoidal category. Colax monoidal structure on a category $\mathcal{A}$ is the same as lax monoidal structure on $\mathcal{A}^{\mathrm{op}}$; it involves an $n$-ary product $\mathcal{A}^{n} \rightarrow \mathcal{A}$ for each $n$. The colax part of the structure consists of maps

$$
a_{1} a_{2} \ldots a_{n} \rightarrow\left(a_{1} \ldots a_{i}\right)\left(a_{i+1} \ldots a_{j}\right) \ldots\left(a_{k+1} \ldots a_{n}\right)
$$

going into a product of products, from the expanded product; these are of course subject to various conditions. The case $n=0$ of the product amounts to an object $i \in \mathcal{A}$, and part of the colax structure involves maps $a \rightarrow i a$ and $a \rightarrow a i$. For the connection between colax monoidal categories and multicategories, see [9] or [1]. For applications of lax monoidal structure to higher categories, see [22] and [2]. 
Recently, the notion of skew monoidal category has received a lot of attention, due to Szlachányi's brilliant insight [27] that they can be used to describe bialgebroids. A skew monoidal category is a category $\mathcal{A}$ equipped with a binary product $\mathcal{A}^{2} \rightarrow \mathcal{A}$ and a unit object $i$, together with natural transformations

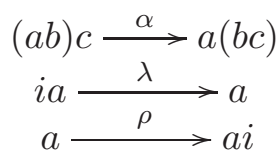

subject to five axioms corresponding to Mac Lane's five axioms for a monoidal category 23. The theory of skew monoidal categories has been developed in a series of papers [15, 18, 17, 19, 16, by the second named author in collaboration with Street.

In 4 the first named author described examples of skew monoidal categories arising from a rather different source - 2-category theory - and used these to give efficient constructions of various more complex monoidal bicategories. In these 2-categorical examples the skew structures were shown to arise from multicategories with two types of multimorphism - one stricter, one weaker.

The main goal of the present paper is to describe a perfect correspondence between skew monoidal categories and a kind of multicategory with two types of multimorphism; unsurprisingly, we call these skew multicategories.

The starting point is the fact that, for a skew monoidal category $\mathcal{A}$, the functor $\mathcal{A} \rightarrow \mathcal{A}$ given by tensoring on the left with the unit object $i$ is a comonad (with counit $\lambda$ ). The category $\mathcal{A}$ therefore comes equipped with a notion of "weak morphism" from $a$ to $b$, consisting of a morphism in the usual sense from $i a$ to $b$. These can be composed as in the Kleisli category of the comonad. But there are also "weak multimorphisms", classified by products such as $(i a) b$ or $((i a) b) c$, as well as a stricter sort of multimap classified by products such $a b$ or $(a b) c$.

It turns out that the resulting notion of skew multicategory is controlled by a particular (non-symmetric) Cat-enriched operad $\mathcal{R}$, and it is convenient to develop the general notion of $\mathcal{T}$-multicategory for a Cat-operad $\mathcal{T}$; then skew multicategories are $\mathcal{R}$-multicategories, and ordinary multicategories are $\mathcal{N}$-multicategories, where $\mathcal{N}$ is the terminal Cat-operad. These $\mathcal{T}$-multicategories can be described using existing notions of generalised multicategory, like that of [7]. The various approaches are described in more detail in Section 3 ,

Just as for ordinary monoidal categories and multicategories, various aspects of skew monoidal categories can most easily be seen from the point of view of skew multicategories. In a future paper [6], we shall study braidings and symmetries for skew monoidal categories. If one thinks of these as extra structure borne by the tensor product, one might be led to write down an overly simplistic definition, but from the multicategorical point of view things become far clearer.

We now outline the structure of the current paper. After a brief review of multicategories and operads, we begin with the notion of $\mathcal{T}$-multicategory in Section 3 and discuss representability in this context. In Section 4 we define skew multicategories as $\mathcal{R}$-multicategories for a Cat-operad $\mathcal{R}$, giving 
examples and defining left representable skew multicategories. In Section 5 we define colax $\mathcal{T}$-algebras, relate them to $\mathcal{T}$-multicategories, and identify those corresponding to left representable skew multicategories.

Using this, as well as results from our companion paper [5, we describe in Section 6 the perfect correspondence between skew monoidal categories and left representable skew multicategories. In Section 6 we also describe variants of this correspondence, dealing with closed skew monoidal categories and skew closed categories.

Acknowledgements. Both authors acknowledge with gratitude the support of an Australian Research Council Discovery Grant DP130101969; Lack further acknowledges the support of a Future Fellowship FT110100385.

\section{REVIEW OF MUlticAtegories AND OPERADS}

In this section we briefly review the definitions, establishing our terminology along the way.

In this paper our operads will always be of the "plain" variety, without actions of the symmetric groups. On the other hand, they will usually be enriched over Cat. Such plain Cat-enriched operads can be equivalently be described as clubs over $\mathbb{N}[14$.

2.1. Multicategories. A multicategory $\mathbb{A}$ consists of:

- a collection of objects

- for each (possibly empty) list $a_{1}, \ldots, a_{n}$ of objects and each object $b$, a set $\mathbb{A}\left(a_{1}, \ldots, a_{n} ; b\right)$; sometimes we write $\bar{a}$ for the list, and then $\mathbb{A}(\bar{a} ; b)$ for the set

- for each object $a$, an element $1_{a} \in \mathbb{A}(a ; a)$

- substitution operations

$$
\mathbb{A}\left(b_{1}, \ldots, b_{n} ; c\right) \times \prod_{i=1}^{n} \mathbb{A}\left(\bar{a}_{i} ; b_{i}\right) \rightarrow \mathbb{A}\left(\bar{a}_{1}, \ldots, \bar{a}_{n} ; c\right)
$$

where each $\bar{a}_{i}$ is itself a list $a_{i 1}, \ldots, a_{i k_{i}}$, and where in the codomain these lists have been concatenated to obtain a list $a_{11}, \ldots, a_{n k_{n}}$.

The notation for substitution is $\left(g, f_{1}, \ldots, f_{n}\right) \mapsto g\left(f_{1}, \ldots, f_{n}\right)$. We require the evident associativity conditions as well as the identity laws $1_{c}(g)=g=$ $g\left(1_{b_{1}}, \ldots, 1_{b_{n}}\right)$.

Remark 2.1. Given $g \in \mathbb{A}\left(b_{1}, \ldots, b_{n} ; c\right)$ and $f: \bar{a} \rightarrow b_{i}$ we sometimes write $g \circ_{i} f$ for the multimap $g(1, \ldots, 1, f, 1, \ldots, 1)$ which captures substitution in position $i$. In the present paper we will use $g \circ_{i} f$ only to simplify notation but note that the notion of multicategory can be formulated with these operations as the primitive ones (see 25] for the operad case).

In a Cat-enriched multicategory each $\mathbb{A}\left(a_{1}, \ldots, a_{n} ; b\right)$ is now a category, and each of the substitution operations a functor. The equations are required to hold strictly. 
2.2. Operads. When a multicategory has only one object, there is no need to keep track of the objects $a_{1}, \ldots, a_{n}, b$ in $\mathbb{A}\left(a_{1}, \ldots, a_{n} ; b\right)$ and so one may simply write $\mathbb{A}_{n}$, except that we typically use names like $\mathcal{T}$ rather than $\mathbb{A}$ for an operad, and so we would write $\mathcal{T}_{n}$ rather than $\mathbb{A}_{n}$. There is then a unit $e \in \mathcal{T}_{1}$; and substitution operations $\mathcal{T}_{n} \times \mathcal{T}_{k_{1}} \times \ldots \times \mathcal{T}_{k_{n}} \rightarrow \mathcal{T}_{k_{1}+\ldots+k_{n}}$.

We are generally interested in the Cat-enriched case, in which we shall speak of a Cat-operad. Each Cat-operad $\mathcal{T}$ has a dual $\mathcal{T}^{*}$ with $\mathcal{T}_{n}^{*}=\mathcal{T}_{n}^{\text {op }}$, and with the corresponding multiplication and unit. (From the point of view of Cat-enriched multicategories, one might think of this as " $\mathcal{T}$ co", obtained by reversing the 2 -cells but not the 1 -cells.)

\section{3. $\mathcal{T}$-MUlticategories}

Let $\mathcal{T}$ be a fixed Cat-operad; recall that in this paper our operads do not involve actions of the symmetric groups.

Definition 3.1. A $\mathcal{T}$-multicategory $\mathbb{A}$ consists of a set $A$ of objects, together with

- for each list $a_{1}, \ldots, a_{n} \in A$ and each $b \in A$, a functor

$$
\mathbb{A}\left(a_{1}, \ldots, a_{n} ; b\right): \mathcal{T}_{n} \rightarrow \text { Set }
$$

whose value at an object $x \in \mathcal{T}_{n}$ we write as $\mathbb{A}_{x}\left(a_{1}, \ldots, a_{n} ; b\right)$ or sometimes $\mathbb{A}_{x}(\bar{a} ; b)$, where $\bar{a}$ stands for the list $a_{1}, \ldots, a_{n}$;

- for each $a \in A$ an element $1_{a} \in \mathbb{A}_{e}(a ; a)$ called the identity;

- substitution maps

$$
\begin{gathered}
\mathbb{A}_{x}\left(b_{1}, \ldots, b_{n} ; c\right) \times \prod_{i=1}^{n} \mathbb{A}_{x_{i}}\left(\bar{a}_{i} ; b_{i}\right) \longrightarrow \mathbb{A}_{x\left(x_{1}, \ldots, x_{n}\right)}\left(\bar{a}_{1}, \ldots, \bar{a}_{n} ; c\right) \\
\left(g, f_{1}, \ldots f_{n}\right) \longmapsto g\left(f_{1}, \ldots, f_{n}\right)
\end{gathered}
$$

natural in $x, x_{1}, \ldots, x_{n}$

satisfying the associativity and identity axioms which are the natural " $\mathcal{T}$ typed" analogues of those for ordinary multicategories.

Example 3.2. If $\mathcal{T}$ is the terminal Cat-operad $\mathcal{N}$ with $\mathcal{N}_{n}=1$ for all $n$, a $\mathcal{T}$-multicategory is just an ordinary multicategory.

Remark 3.3. In the $\mathcal{T}$-multicategory context we also sometimes write $g \circ_{i} f$ for the multimap $g(1, \ldots, 1, f, 1, \ldots, 1)$. And as in the ordinary setting, $\mathcal{T}$-multicategories admit a formulation taking such operations as primitive.

Just as for ordinary multicategories, every $\mathcal{T}$-multicategory $\mathbb{A}$ has an associated category $\mathcal{A}$ with the same objects, with homs given by $\mathcal{A}(a, b)=$ $\mathbb{A}_{e}(a ; b)$, and with composition given by substitution.

Proposition 3.4. For each $n$, the functor

$$
\mathbb{A}_{-}(-;-): \mathcal{T}_{n} \times\left(A^{n}\right)^{\mathrm{op}} \times A \rightarrow \text { Set }
$$

extends to a functor

$$
\mathbb{A}_{-}(-;-): \mathcal{T}_{n} \times\left(\mathcal{A}^{n}\right)^{\mathrm{op}} \times \mathcal{A} \rightarrow \text { Set. }
$$

These extensions are uniquely determined by the following properties:

(1) $\mathbb{A}_{e}(-,-)=\mathcal{A}(-,-): \mathcal{A}^{o p} \times \mathcal{A} \rightarrow$ Set; 
(2) the substitution maps

$$
\mathbb{A}_{x}\left(b_{1}, \ldots, b_{n} ; c\right) \times \prod_{i=1}^{n} \mathbb{A}_{x_{i}}\left(\bar{a}_{i} ; b_{i}\right) \rightarrow \mathbb{A}_{x\left(x_{1}, \ldots, x_{n}\right)}\left(\bar{a}_{1}, \ldots, \bar{a}_{n} ; c\right)
$$

are natural in each $\bar{a}_{i}, b_{i}$ and $c$ as well as the variables $x, x_{i}$.

Proof. The requirements force us to define $\mathbb{A}_{x}\left(a_{1}, \ldots, a_{n} ;-\right)$ using the substitution map

$$
\mathbb{A}_{e}(b ; c) \times \mathbb{A}_{x}\left(a_{1}, \ldots, a_{n} ; b\right) \longrightarrow \mathbb{A}_{x}\left(a_{1}, \ldots, a_{n} ; c\right)
$$

and in the other variables using the substitution map

$$
\mathbb{A}_{x}\left(b_{1}, \ldots, b_{n} ; c\right) \times \prod_{i=1}^{n} \mathbb{A}_{e}\left(a_{i} ; b_{i}\right) \longrightarrow \mathbb{A}_{x}\left(a_{1}, \ldots, a_{n} ; c\right) .
$$

There is a straightforward way to adapt the notion of morphism of multicategories and 2-cell to our $\mathcal{T}$-dependent context. A morphism of $\mathcal{T}$ multicategories $\mathbb{A} \rightarrow \mathbb{B}$ involves an assignment $a \mapsto F a$ on objects, together with maps

$$
F: \mathbb{A}_{x}\left(a_{1}, \ldots, a_{n} ; b\right) \rightarrow \mathbb{B}_{x}\left(F a_{1}, \ldots, F a_{n} ; F b\right)
$$

which preserve substitution and identities in the obvious sense.

Notation 3.5. When we wish to apply a map $F$ to each element of a list $\bar{a}$, we write $F \bar{a}$. Thus the maps displayed above could be written as

$$
F: \mathbb{A}_{x}(\bar{a} ; b) \rightarrow \mathbb{B}_{x}(F \bar{a} ; F b) .
$$

Given two such morphisms $F$ and $G$, a 2-cell $\varphi: F \rightarrow G$ involves a morphism $\varphi_{a}: F a \rightarrow G a$ in $\mathbb{B}_{e}$ for each $a \in \mathbb{A}$, subject to the naturality condition asserting that the squares

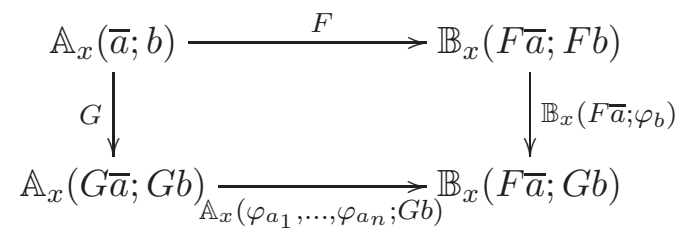

commute.

$\mathcal{T}$-multicategories, their morphisms, and their 2-cells together form a 2category T-Mult.

Definition 3.6. A $\mathcal{T}$-multicategory is weakly representable when each of the functors $\mathbb{A}_{x}\left(a_{1}, \ldots, a_{n} ;-\right): \mathcal{A} \rightarrow$ Set is representable.

Explicitly, this means that for each $x \in \mathcal{T}_{n}$ and each $a_{1}, \ldots, a_{n} \in A$ there exists an object $m_{x}\left(a_{1}, \ldots, a_{n}\right) \in A$ and multimap

$$
\theta_{x}(\bar{a}) \in \mathbb{A}_{x}\left(\bar{a} ; m_{x} \bar{a}\right)
$$

with the property that the induced function

$$
-\circ_{1} \theta_{x}(\bar{a}): \mathbb{A}_{e}\left(m_{x} \bar{a} ; b\right) \rightarrow A_{x}(\bar{a} ; b)
$$

is a bijection for all $b \in A$. We sometimes call $\theta_{x}(\bar{a})$ a universal multimap of type $x$. 
Remark 3.7. In the case that $\mathcal{T}$ is the terminal Cat-operad there is a single object $m\left(a_{1}, \ldots, a_{n}\right)$ for each $n$. A weakly representable multicategory is representable [12] when substitution with $\theta(\bar{a})$ induces bijections

$$
\mathbb{A}(\bar{b}, m \bar{a}, \bar{c} ; d) \rightarrow \mathbb{A}(\bar{b}, \bar{a}, \bar{c} ; d) .
$$

It is possible to make a corresponding definition of representable $\mathcal{T}$-multicategory for general $\mathcal{T}$; we do not do so, since in the case $\mathcal{T}=\mathcal{R}$ corresponding to skew multicategories this is not the representability condition which captures the notion of skew monoidal category. In fact for general $\mathcal{T}$, the only representability condition we consider is that of weak representability.

Returning to the case of a general $\mathcal{T}$ and a weakly representable $\mathcal{T}$ multicategory $\mathbb{A}$, by Proposition 3.4 we have functors

$$
\mathbb{A}_{-}(-, \ldots,-;-): \mathcal{T}_{n} \times\left(\mathcal{A}^{n}\right)^{\mathrm{op}} \times \mathcal{A} \rightarrow \text { Set }
$$

which are representable in the last variable, so in the usual way there is an induced functor

$$
\mathcal{T}_{n}^{\text {op }} \times \mathcal{A}^{n} \rightarrow \mathcal{A}
$$

whose action on objects we may write as $\left(x, a_{1}, \ldots, a_{n}\right) \mapsto m_{x}\left(a_{1}, \ldots, a_{n}\right)$, together with bijections

$$
\mathcal{A}\left(m_{x}\left(a_{1}, \ldots, a_{n}\right), b\right) \cong \mathbb{A}_{x}\left(a_{1}, \ldots, a_{n} ; b\right)
$$

natural in all variables $x, a_{1}, \ldots, a_{n}, b$.

These functors $\mathcal{T}_{n}^{\mathrm{op}} \times \mathcal{A}^{n} \rightarrow \mathcal{A}$ can in turn be thought of as functors

$$
m: \mathcal{T}_{n}^{\text {op }} \rightarrow\left[\mathcal{A}^{n}, \mathcal{A}\right]
$$

where $\left[\mathcal{A}^{n}, \mathcal{A}\right]$ represents the functor category. The category $\mathcal{A}$ together with the functors $m$ capture much of the structure of the $\mathcal{T}$-multicategory: the set of objects, the various multihoms, the identities, and a few special cases of the substitutions. We shall see in Section 5 that the remaining structure can be understood in terms of colax algebras.

Proposition 3.8. For a weakly representable $\mathcal{T}$-multicategory $\mathbb{A}$, it is possible to choose the corresponding functor $m: \mathcal{T}_{1}^{\mathrm{op}} \rightarrow[\mathcal{A}, \mathcal{A}]$ to send the unit $e \in \mathcal{T}_{1}^{\mathrm{op}}$ to the identity functor.

Proof. The functor $m_{e}: \mathcal{A} \rightarrow \mathcal{A}$ is characterized by the fact that it provides representing objects for the Yoneda embedding $\mathcal{A} \rightarrow\left[\mathcal{A}^{\mathrm{op}}\right.$, Set $]$, in the sense that there are natural isomorphisms $\mathcal{A}\left(m_{e}(a), b\right) \cong \mathcal{A}(a, b)$, so clearly we may take it to be the identity.

There are various other perspectives on $\mathcal{T}$-multicategories, not needed for this paper, but which may help to shed light on the concept. We begin with the simplified case of a Set-operad by way of motivation.

Alternative perspective 1 . If $\mathcal{T}$ is a Set-operad we can view it as a Cat-operad in which each category $\mathcal{T}_{n}$ is discrete. Then a $\mathcal{T}$-multicategory amounts to an ordinary multicategory equipped with a multifunctor into $\mathcal{T}$, itself viewed as a one object multicategory.

The Set-operad gives rise to a cartesian monad on Set; one can then define generalised multicategories relative to this cartesian monad - for instance, see 22 - and these coincide with our $\mathcal{T}$-multicategories. 
Furthermore the monad on Set extends to a monad $\mathcal{T}$ on the pseudodouble category of spans, and a $\mathcal{T}$-multicategory in our sense is then the same as a $\mathcal{T}$-monoid in the sense of [7.

Alternative perspective 2. Now suppose that $\mathcal{T}$ is a general Cat-operad. If $\mathbb{A}$ is a $\mathcal{T}$-multicategory, there is an associated Cat-enriched multicategory $\overline{\mathbb{A}}$ with the same objects. The multihom $\overline{\mathbb{A}}\left(a_{1}, \ldots, a_{n} ; b\right)$ is the category of elements of the functor $\mathbb{A}\left(a_{1}, \ldots, a_{n} ; b\right): \mathcal{T}_{n} \rightarrow$ Set. The substitutions for $\mathbb{A}$ induce the necessary substitutions for $\overline{\mathbb{A}}$. The projections $\overline{\mathbb{A}}\left(a_{1}, \ldots, a_{n} ; b\right) \rightarrow \mathcal{T}_{n}$ are of course discrete opfibrations, and they define a Cat-enriched multifunctor from $\overline{\mathbb{A}}$.

This provides an alternative characterization of $\mathcal{T}$-multicategories, as the Cat-enriched multicategories in the usual sense, equipped with a Catenriched multifunctor into $\mathcal{T}$ which is locally a discrete opfibration. Here when we speak of "multifunctor into $\mathcal{T}$ ", we are again regarding the operad $\mathcal{T}$ as a one-object multicategory. This in turn makes it clear that one could define $\mathcal{T}$-multicategories for any Cat-enriched multicategory $\mathcal{T}$, not just an operad.

A Cat-operad $\mathcal{T}$ gives rise to a 2-monad on Cat which extends to a monad $\mathbb{T}$ on the pseudo-double category of categories and profunctors; now a $\mathcal{T}$-multicategory in our sense is the same as a $\mathbb{T}^{*}$-monoid [7] with discrete underlying category, where $\mathcal{T}^{*}$ is the dual operad.

Alternative perspective 3. There is yet another possible characterization, which is relevant to what follows: a colax $\mathcal{T}$-algebra in the monoidal bicategory Mat of Set-valued matrices. An object of Mat is a set $A$. A morphism from $A$ to $B$ is an $(A \times B)$-indexed family of sets, and a 2-cell is an $(A \times B)$-indexed family of functions. Morphisms are composed using the usual formula for matrix multiplication.

For each set $A$ there is a (pseudo) Cat-enriched operad $\operatorname{End}(A)$, with $\operatorname{End}(A)_{n}=\operatorname{Mat}\left(A^{n}, A\right)$, and a colax $\mathcal{T}$-algebra is a colax morphism of operads from $\mathcal{T}$ to $\operatorname{End}(A)$. This involves a functor $\mathcal{T}_{n} \rightarrow \operatorname{Mat}\left(A^{n}, A\right)$ for each $n$, sending $x \in \mathcal{T}_{n}$ to the family $\mathbb{A}_{x}$ whose component indexed by $\left(a_{1}, \ldots, a_{n}\right)$ and $b$ is $\mathbb{A}_{x}\left(a_{1}, \ldots, a_{n} ; b\right)$. The colax structure is given by the substitution and identity maps.

\section{Skew multicategories}

In this section we specialize to a particular Cat-operad $\mathcal{R}$, and define skew multicategories to be $\mathcal{R}$-multicategories. It turns out that the (strict) $\mathcal{R}$-algebras are precisely the skew monoidal categories for which the associativity maps $\alpha$ and the left unit maps $\lambda$ are identities, although we shall never need to use this fact.

We define $\mathcal{R}$ explicitly as follows

$$
\mathcal{R}_{n}= \begin{cases}\{\ell\} & \text { if } n=0 \\ \{\lambda: t \rightarrow \ell\} & \text { otherwise }\end{cases}
$$

so that abstractly $\mathcal{R}_{n}$ is the arrow category $\mathbf{2}$ for $n>0$ and $\mathcal{R}_{0}$ is the terminal category 1 . 
The multiplication $\mathcal{R}_{n} \times \mathcal{R}_{k_{1}} \times \ldots \times \mathcal{R}_{k_{n}} \rightarrow \mathcal{R}_{k_{1}+\ldots+k_{n}}$ is defined by

$$
x\left(x_{1} \ldots, x_{n}\right)= \begin{cases}t & \text { if } x=x_{1}=t \\ \ell & \text { otherwise }\end{cases}
$$

and the unit by $t \in \mathcal{R}_{1}$.

Remark 4.1. For an object or morphism $x$ in $\{\lambda: t \rightarrow \ell\}$ we sometimes write $x_{n}$ when we are thinking of it as lying in $\mathcal{R}_{n}$.

Definition 4.2. A skew multicategory is an $\mathcal{R}$-multicategory.

Let us unpack the definition. To begin with, a skew multicategory involves

- a set of objects $A$

- for each $a \in A$ a set $\mathbb{A}_{\ell}(; a)$ of nullary maps

- for each $n>0$, each $a_{1}, \ldots, a_{n} \in A$, and each $b \in A$ a function

$$
j_{\bar{a}, b}: \mathbb{A}_{t}\left(a_{1}, \ldots, a_{n} ; b\right) \rightarrow \mathbb{A}_{\ell}\left(a_{1}, \ldots, a_{n} ; b\right) .
$$

We sometimes refer to the elements of $\mathbb{A}_{t}\left(a_{1}, \ldots, a_{n} ; b\right)$ as tight $n$-ary multimaps, and to the elements of $\mathbb{A}_{\ell}\left(a_{1}, \ldots, a_{n} ; b\right)$ as loose $n$-ary multimaps. The functions (4.1) then allow us to view each tight multimap as a loose multimap.

On top of this there is further structure:

- for each $a \in A$ there is a tight multimap $1_{a} \in \mathbb{A}_{t}(a ; a)$;

- substitution gives us multimaps $g\left(f_{1}, \ldots, f_{n}\right)$, which are tight just when $g$ and $f_{1}$ are; these substitutions, moreover, commute with the comparisons viewing tight multimaps as loose.

Finally the usual associativity and unit axioms must be satisfied.

In many of our leading examples of skew multicategories the functions (4.1) are subset inclusions. In that case we can view skew multicategories as ordinary multicategories equipped with a distinguished class of tight multimaps, as we now record.

Proposition 4.3. There is a bijection between

(1) Skew multicategories $\mathbb{A}$ in which each

$$
j_{\bar{a}, b}: \mathbb{A}_{t}\left(a_{1}, \ldots, a_{n} ; b\right) \rightarrow \mathbb{A}_{\ell}\left(a_{1}, \ldots, a_{n} ; b\right)
$$

is a subset inclusion, and

(2) Multicategories $\mathbb{A}$ together with for each $n>0$ specified subsets

$$
\mathbb{A}_{t}\left(a_{1}, \ldots, a_{n} ; b\right) \subseteq \mathbb{A}\left(a_{1}, \ldots, a_{n} ; b\right)
$$

of "tight" maps containing the identities and having the property that a composite multimap $g\left(f_{1}, \ldots, f_{n}\right)$ is tight whenever both $g$ and $f_{1}$ are tight.

4.1. Skew multicategories versus ordinary multicategories. There is a forgetful 2-functor

$$
U: \text { R-Mult } \rightarrow \text { Mult }
$$

sending a skew multicategory $\mathbb{A}$ to the multicategory $\mathbb{A}_{\ell}$ with the same objects and sets of multimaps $\mathbb{A}_{\ell}(\bar{a} ; b)$. Its underlying ordinary functor has a left adjoint, which views a multicategory as a skew multicategory in which only the identities are tight. $U$ also has a right 2 -adjoint which views a 
multicategory as a skew multicategory in which all multimorphisms are tight. We can identify multicategories with skew multicategories satisfying either condition; the all multimorphisms are tight identification has the advantage of extending not only to 1-cells but 2-cells too.

Since $\mathbb{A}_{\ell}$ is a multicategory it has an underlying category $\mathcal{A}_{\ell}$ with the same objects as $\mathbb{A}$ and with morphisms the loose unary maps. The components

$$
j_{a, b}: \mathbb{A}_{t}(a ; b) \rightarrow \mathbb{A}_{\ell}(a ; b)
$$

then give the action on morphisms of an identity on objects functor $j: \mathcal{A} \rightarrow$ $\mathcal{A}_{\ell}$, which often has a left adjoint - see Proposition 6.2.

4.2. 2-categorical examples. Although no definition of skew multicategory was given in 4, various "2-categorical" examples were given there and in [13. These are perhaps best introduced via a simple example. (Each of these examples is, in fact, a Cat-enriched skew multicategory but we will not treat the enrichment here.)

Let $\mathbb{F P}$ denote the multicategory whose objects are categories equipped with a choice of finite products. For $n>0$ a multimap is a functor $F: \mathcal{A}_{1} \times$ $\ldots \times \mathcal{A}_{n} \rightarrow \mathcal{B}$ preserving products in each variable in the usual up to isomorphism sense. A nullary map, an element of $\mathbb{F P}(; \mathcal{B})$, is an object of $\mathcal{B}$. Substitution is defined in the usual way and the multicategory axioms are routinely verified.

We declare a multimap $F$ as above to be tight just when it preserves the given products strictly in the first variable; that is, when each functor $F\left(-, a_{2}, \ldots, a_{n}\right): \mathcal{A}_{1} \rightarrow \mathcal{B}$ preserves the given products strictly. These tight morphism are easily seen to be closed under substitution in the first variable, and the complete structure therefore forms a skew multicategory.

One can modify this in various ways; for example, there is a skew multicategory $\mathbb{A}$ with the same objects as $\mathbb{F} \mathbb{P}$ in which $\mathbb{A}_{\ell}\left(\mathcal{A}_{1}, \ldots, \mathcal{A}_{n} ; \mathcal{B}\right)$ is given by arbitrary functors $\mathcal{A}_{1} \times \ldots \times \mathcal{A}_{n} \rightarrow \mathcal{B}$, and the tight maps are those which preserve finite products (in the usual sense) in the first variable.

More generally, as proven in [13, for any pseudo-commutative 2-monad $T$ on Cat, there is a multicategory whose objects are the (strict) $T$-algebras, and whose multimaps are the functors $F: \mathcal{A}_{1} \times \ldots \times \mathcal{A}_{n} \rightarrow \mathcal{B}$ equipped with the structure of an algebra pseudomorphism in each variable separately, with these $n$ pseudomorphism structures satisfying certain compatibility conditions. The tight morphisms, once again defined to be those which are strict in the first variable, are closed under substitution so that the complete structure forms a skew multicategory.

For instance, one could take $T$ to be the 2-monad for symmetric strict monoidal categories (also known as permutative categories). The corresponding multicategory (of loose maps) was defined in [11, Definition 3.1]. Or one could replace permutative categories by symmetric monoidal categories, braided monoidal categories, or categories with chosen limits or colimits of some given class.

4.3. Skew monoidal categories as skew multicategories. In Section6.1 we will see that any skew monoidal category $\mathcal{C}$ gives rise to a skew multicategory $\mathbb{C}$, and that the resulting skew multicategories are precisely the left representable ones, to which we now turn. 
4.4. Left representability. By Definition 3.6, a skew multicategory $\mathbb{A}$ is weakly representable if for each pair $x \in \mathcal{R}_{n}$ and $\bar{a} \in A^{n}$ there exists an object $m_{x} \bar{a} \in A$ and multimap

$$
\theta_{x}(\bar{a}) \in \mathbb{A}_{x}\left(\bar{a} ; m_{x} \bar{a}\right)
$$

with the property that the induced function

$$
-\circ_{1} \theta_{x}(\bar{a}): \mathbb{A}_{t}\left(m_{x} \bar{a} ; b\right) \rightarrow \mathbb{A}_{x}(\bar{a} ; b)
$$

is a bijection for all $b \in A$.

Observe that for all $x \in \mathcal{R}_{n}$ we have the equation $x_{n+m-1}=t_{m} \circ_{1} x_{n}$. Therefore a multimap $\theta_{x}(\bar{a})$ as above induces for each $\bar{b} \in A^{m}, c \in A$ a function

$$
-\circ_{1} \theta_{x}(\bar{a}): \mathbb{A}_{t}\left(m_{x} \bar{a}, \bar{b} ; c\right) \rightarrow \mathbb{A}_{x}(\bar{a}, \bar{b} ; c)
$$

In the case that the above function is invertible for all $\bar{b}, c$ as above we say that $\theta_{x}(\bar{a})$ is left universal.

Definition 4.4. Let $\mathbb{A}$ be a weakly representable skew multicategory. We say that $\mathbb{A}$ is left representable if the function (4.2) is a bijection for all $x, \bar{a}, \bar{b}$ and $c$, and all universal multimaps $\theta_{x}(\bar{a})$.

Let $\mathbf{S k e}_{\ell}$ be the 2-category of skew monoidal categories, (lax) monoidal functors, and monoidal natural transformations. The following theorem, proved in Section 6, is the main result of the present paper. It is the skew analogue of Theorem 9.8 of [12].

Theorem 6.1. There is a 2-equivalence between the 2-category $\mathbf{S k w}_{\ell}$ and the full sub 2-category of $\mathbf{R}$-Mult consisting of the left representable skew multicategories.

In order to obtain a better understanding of left representability, we isolate two classes of universal multimap. First, we refer to a universal multimap

$$
\theta_{\ell_{0}} \in \mathbb{A}_{\ell}\left(; m_{\ell_{0}}\right)
$$

as a nullary map classifier; and second, we refer to a universal multimap

$$
\theta_{t}\left(a_{1}, a_{2}\right) \in \mathbb{A}_{t}\left(a_{1}, a_{2} ; m_{t}\left(a_{1}, a_{2}\right)\right)
$$

as a tight binary map classifier.

Given these two classes of multimaps we can construct objects and multimaps

$$
\theta_{x}(\bar{a}) \in \mathbb{A}_{x}\left(\bar{a} ; m_{x} \bar{a}\right)
$$

for all $x, \bar{a}$ using the inductive formulae

$$
\begin{gathered}
m_{t_{1}}(a)=a \text { and } \theta_{t_{1}}(a)=1_{a} \in \mathbb{A}_{t}(a ; a) \\
m_{x_{n+1}}\left(a_{1}, \ldots, a_{n}, a_{n+1}\right)=m_{t}\left(m_{x_{n}}\left(a_{1}, \ldots, a_{n}\right), a_{n+1}\right)
\end{gathered}
$$

and

$$
\theta_{x_{n+1}}\left(a_{1}, \ldots, a_{n}, a_{n+1}\right)=\theta_{t}\left(m_{x}\left(a_{1}, \ldots, a_{n}\right), a_{n+1}\right) \circ_{1} \theta_{x_{n}}\left(a_{1}, \ldots, a_{n}\right)
$$

Proposition 4.5. Let $\mathbb{A}$ be a skew multicategory. The following are equivalent:

(1) $\mathbb{A}$ is left representable; 
(2) $\mathbb{A}$ admits tight binary map classifiers and a nullary map classifier and the multimaps $\theta_{x}(\bar{a})$ constructed from these according to (4.3), (4.4) and (4.5) are universal;

(3) $\mathbb{A}$ admits tight binary map classifiers and a nullary map classifier and these are left universal;

(4) $\mathbb{A}$ is weakly representable and for all $b, c \in A$ the functions

$$
-\circ_{1} \theta_{x}(\bar{a}): \mathbb{A}_{t}\left(m_{x} \bar{a}, b ; c\right) \rightarrow \mathbb{A}_{x}(\bar{a}, b ; c)
$$

are invertible.

Proof. By definition of left representability $(1 \Longrightarrow 3,4)$.

Suppose that $\mathbb{A}$ admits tight binary and nullary map classifiers and consider the multimaps $\theta_{x}(\bar{a})$ constructed as in (4.3), (4.4) and (4.5). Associativity then gives a commutative triangle

$$
\begin{aligned}
& \mathbb{A}_{t}\left(m_{t}\left(m_{x}(\bar{a}), b\right), \bar{c} ; d\right) \\
-\circ_{1} \theta_{t}\left(m_{x}(\bar{a}), b\right) & \downarrow \\
\mathbb{A}_{t}\left(m_{x}(\bar{a}), b, \bar{c} ; d\right) \stackrel{-\circ_{1} \theta_{x}(\bar{a}, b)}{\longrightarrow} \mathbb{A}_{x}(\bar{a}, b, \bar{c} ;(\bar{a}) & \longrightarrow
\end{aligned}
$$

for all tuples $\bar{a}, \bar{c}$ and objects $b, d$.

Let us show that $(2 \Longrightarrow 1)$. We must show that $-\circ_{1} \theta_{x}(\bar{a}): \mathbb{A}_{x}\left(m_{x}(\bar{a}), \bar{c} ; d\right) \rightarrow$ $\mathbb{A}_{x}(\bar{a}, \bar{c} ; d)$ is invertible for all for all $x, \bar{a}$ and tuples $\bar{c}$ of length $n$. The case $n=0$ is our hypothesis. For the inductive step, consider $(b, \bar{c})$ as a generic $(n+1)$-tuple; we must show that the horizontal leg above is invertible. But both the vertical and diagonal cases are invertible by the inductive hypothesis; hence the horizontal leg is so too.

Let us prove that $(4 \Longrightarrow 2)$. By assumption we certainly have tight binary and nullary map classifiers. Furthermore the identities 1: $a \rightarrow a$ of (4.3) are always $t_{1}$-universal. It remains to prove that if (4) holds then the inductive constructions of (4.4) and (4.5) preserve universality. That is, we must prove that the composite

$$
\begin{aligned}
& \mathbb{A}_{t}\left(m_{t}\left(m_{x}(\bar{a}), a_{n+1}\right) ; b\right) \\
& -\circ_{1} \theta_{t}\left(m_{x}(\bar{a}), a_{n+1}\right) \mid \\
& \mathbb{A}_{t}\left(m_{x}(\bar{a}), a_{n+1} ; b\right) \underset{-\circ_{1} \theta_{x}(\bar{a})}{\longrightarrow} \mathbb{A}_{x}\left(\bar{a}, a_{n+1} ; b\right)
\end{aligned}
$$

is invertible. The horizontal map is invertible by universality, and the vertical is invertible by universality and the assumption in (4).

Finally we prove that $(3 \Longrightarrow 2)$. For this the basic and evident observation is that if $f \in \mathbb{A}_{x}(\bar{a} ; b)$ and $g \in \mathbb{A}_{t}(b, c ; d)$ are both left universal then so is $g \circ_{1} f \in \mathbb{A}_{x}(\bar{a}, b, c ; d)$. Now the identities $1: a \rightarrow a$ of (4.3) are always left universal as are the given multimaps in the nullary and binary case by assumption; since the maps $\theta_{x}(\bar{a})$ are obtained from these by composition as above these are always left universal too.

\subsection{Closed skew multicategories with unit.}


Definition 4.6. A skew multicategory $\mathbb{A}$ is said to be closed if for all $b, c \in \mathbb{A}$ there exists an object $[b, c]$ and tight multimap $e_{b, c} \in \mathbb{A}_{t}([b, c], b ; c)$ with the universal property that the induced function

$$
e_{b, c} \circ_{1}-: \mathbb{A}_{x}\left(a_{1}, \ldots, a_{n} ;[b, c]\right) \rightarrow \mathbb{A}_{x}\left(a_{1}, \ldots, a_{n}, b ; c\right)
$$

is a bijection for all $a_{1}, \ldots, a_{n} \in A$ and $x \in \mathcal{R}_{n}$.

If a closed skew multicategory admits a nullary map classifier, then we call it a closed skew multicategory with unit.

Let $\mathbf{S k e w}_{\ell} \mathbf{C l}_{\ell}$ be the 2-category of skew closed categories [26], closed functors and closed natural transformations. In Section 6 we prove the following theorem.

Theorem 6.6. There is a 2-equivalence between the 2-category $\mathbf{S k e w} \mathbf{C l}_{\ell}$ and the full sub 2-category of R-Mult consisting of the closed skew multicategories with unit.

We will need one element of the above correspondence in the next section. By (4.7) we have isomorphisms

$$
e_{b, c} \circ_{1}-: \mathbb{A}_{t}(a ;[b, c]) \rightarrow \mathbb{A}_{t}(a, b ; c)
$$

and these are natural in $a$. By Proposition 3.4 the right hand side is a functor $\left(\mathcal{A}^{2}\right)^{o p} \times \mathcal{A} \rightarrow$ Set whence by Yoneda the objects $[b, c]$ extend uniquely to a functor $[-,-]: \mathcal{A}^{o p} \times \mathcal{A} \rightarrow \mathcal{A}$ for which the isomorphisms are also natural in $b$ and $c$. In particular, for each $b \in A$ we have a functor $[b,-]: \mathcal{A} \rightarrow \mathcal{A}$.

4.6. More on left representability and closedness. Combining the above cases, let $\mathbf{S k e w M C l} \mathbf{M C}_{\ell}$ be the 2-category of closed skew monoidal categories, lax monoidal functors and monoidal transformations. In Section 6 we prove:

Theorem6.4. The 2-equivalence of Theorem 6.1 restricts to a 2-equivalence between the 2-category $\mathbf{S k e w} \mathbf{M C l} \mathbf{l}_{\ell}$ of closed skew monoidal categories and the full sub-2-category of $\mathbf{R}$-Mult consisting of the left representable closed skew multicategories.

In general left representability is a stronger condition than weak representability, but if the skew multicategory is closed then the two notions coincide, as the following result shows.

Proposition 4.7. For a closed skew multicategory $\mathbb{A}$, the following are equivalent:

(1) $\mathbb{A}$ is left representable;

(2) $\mathbb{A}$ is weakly representable;

(3) $\mathbb{A}$ admits a nullary map classifier and tight binary map classifiers;

(4) $\mathbb{A}$ admits a nullary map classifier and each functor $[b,-]: \mathcal{A} \rightarrow \mathcal{A}$ has a left adjoint.

Proof. We will show that in a closed skew multicategory any universal multimap $\theta_{x}(\bar{a})$ is left universal. The equivalence of $(1)$ and $(2)$ is then immediate, whilst the equivalence of (1) and (3) then follows from Proposition 4.5. We must prove that for all tuples $\bar{b}$ the function

$$
-\circ_{1} \theta_{x}(\bar{a}): \mathbb{A}_{t}\left(m_{x} \bar{a}, \bar{b} ; c\right) \rightarrow \mathbb{A}_{x}(\bar{a}, \bar{b} ; c)
$$


is invertible for all $c$. We argue by induction on the length $n$ of $\bar{b}$. The case $n=0$ is assumed. Observe that the following diagram commutes.

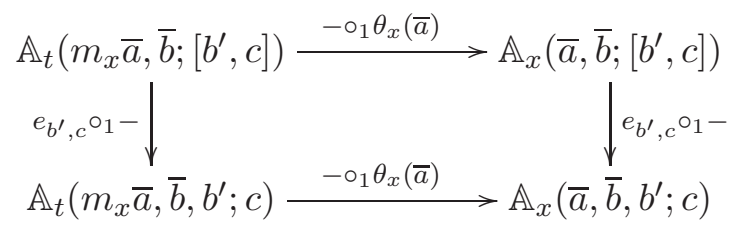

Therefore the invertibility of the vertical morphisms (by closedness) and the top horizontal morphism (by induction) ensure the invertibility of the bottom horizontal morphism, as required.

To prove $(3 \Longleftrightarrow 4)$ it suffices to show that each $[b,-]$ admits a left adjoint if and only if $\mathbb{A}$ admits tight binary map classifiers. The first condition is equivalent to asking for a functor $\otimes: \mathcal{A}^{2} \rightarrow \mathcal{A}$ and isomorphisms $\mathcal{A}(a \otimes b, c) \cong$ $\mathcal{A}(a,[b, c])$ natural in each variable. The second is equivalent to asking for a functor $m_{t}: \mathcal{A}^{2} \rightarrow \mathcal{A}$ and isomorphisms $\mathcal{A}\left(m_{t}(a, b), c\right) \cong \mathbb{A}_{t}(a, b ; c)$ natural in each variable. Since we have isomorphisms $\mathcal{A}(a,[b, c]) \cong \mathbb{A}_{t}(a, b ; c)$ natural in each variable the result follows.

Examples 4.8. Each of the 2-categorical skew multicategories described in 4.2 is associated to an accessible pseudocommutative 2-monad on Cat. Such skew multicategories are both left representable and closed.

The results required to establish these claims are contained in Section 6 of [4]. Briefly, closedness goes back to Theorem 11 of [13], the nullary map classifier is the free $T$-algebra on 1 whilst Proposition 6.3 of [4] establishes that each $[A,-]$ has a left adjoint. Accordingly such skew multicategories are left representable by Proposition 4.7 above.

\section{Colax $\mathcal{T}$-algebras and $\mathcal{T}$-multicategories}

In this section we define colax $\mathcal{T}$-algebras for a Cat-operad $\mathcal{T}$.

Definition 5.1. A colax $\mathcal{T}$-algebra is a category $\mathcal{A}$ together with

- functors $m_{n}: \mathcal{T}_{n} \times \mathcal{A}^{n} \rightarrow \mathcal{A}$ whose value at $\left(x,\left(a_{1}, \ldots, a_{n}\right)\right)$ we denote by $m_{x}\left(a_{1}, \ldots, a_{n}\right)$;

- morphisms $p_{a}: m_{e}(a) \rightarrow a$ natural in $a$;

- substitution maps

$$
m_{x\left(x_{1}, \ldots, x_{n}\right)}\left(\bar{a}_{1}, \ldots, \bar{a}_{n}\right) \stackrel{\Gamma_{x_{1}, \ldots, x_{n}, x}}{\longrightarrow} m_{x}\left(m_{x_{1}}\left(\bar{a}_{1}\right), \ldots, m_{x_{n}}\left(\bar{a}_{n}\right)\right)
$$

natural in all variables $x, x_{i}, \bar{a}_{i}$

satisfying the associativity and identity axioms which are the natural " $\mathcal{T}$ typed" analogues of those for colax monoidal categories: see [22, Definition 3.1.1], for example, for the dual case.

Example 5.2. If $\mathcal{T}$ is the terminal Cat-operad $\mathcal{N}$ with $\mathcal{N}_{n}=1$ for all $n$, a colax- $\mathcal{T}$-algebra is a colax monoidal category.

Definition 5.3. A colax $\mathcal{T}$-algebra is said to be normal if the morphisms $p_{a}: m_{e}(a) \rightarrow a$ are identities. 
Remark 5.4. We will be interested primarily in normal colax $\mathcal{T}$-algebras, since these are the ones corresponding to $\mathcal{T}$-multicategories. In the context of a normal colax $\mathcal{T}$-algebra we obtain substitution maps

$$
m_{x \circ_{i} y}\left(a_{1}, \ldots, a_{i-1}, \bar{b}, a_{i+1}, \ldots, a_{n}\right) \stackrel{\Gamma_{y, i, x}}{\longrightarrow} m_{x}\left(a_{1}, \ldots, a_{i-1}, m_{y}(\bar{b}), a_{i+1}, \ldots, a_{n}\right)
$$

as a special case of (5.1) on setting $x_{j}=1$ for $j \neq i$ and $x=y$.

A lax morphism of colax $\mathcal{T}$-algebras $A \rightarrow B$ involves a functor $F: \mathcal{A} \rightarrow \mathcal{B}$ together with natural families of maps

$$
\tilde{F}_{x, \bar{a}}: m_{x}\left(F a_{1}, \ldots, F a_{n}\right) \rightarrow F m_{x}\left(a_{1}, \ldots, a_{n}\right)
$$

commuting with substitution and identities in the obvious sense.

Given two such lax morphisms $F$ and $G$, a 2-cell $\varphi: F \rightarrow G$ is a natural transformation with the property that the square

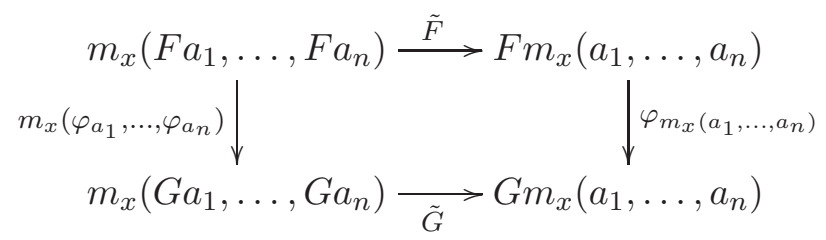

Normal colax $\mathcal{T}$-algebras and their morphisms and 2-cells together form a 2-category $\mathbf{n C o l a x}-\mathbf{T}$ - $\mathbf{A} \lg _{\ell}$.

Recall that $\mathcal{T}^{*}$ denotes the operad obtained from $\mathcal{T}$ by replacing each $\mathcal{T}_{n}$ by $\mathcal{T}_{n}^{\mathrm{op}}$.

Theorem 5.5. There is a fully faithful 2-functor $\mathbf{n C o l a x}-\mathbf{T}^{*}-\mathbf{A} \lg _{\ell} \rightarrow \mathbf{T}-\mathbf{M u l t}$ whose essential image consists of the weakly representable $\mathcal{T}$-multicategories.

Proof. We shall go through the structure involved in a weakly representable $\mathcal{T}$-multicategory and see that it corresponds to that of a normal colax $\mathcal{T}^{*}$ algebra, and that this correspondence respects the various notions of morphism and 2-cell.

First of all, as observed in Proposition 3.4 above, a $\mathcal{T}$-multicategory $\mathbb{A}$ determines a category $\mathcal{A}$ with the same objects, and functors

$$
\mathcal{T}_{n} \times\left(\mathcal{A}^{n}\right)^{\mathrm{op}} \times \mathcal{A} \rightarrow \text { Set }
$$

and so, in the representable case, functors

$$
\mathcal{T}_{n}^{\mathrm{op}} \times \mathcal{A}^{n} \rightarrow \mathcal{A} \text {. }
$$

By Proposition 3.8, we may take $m_{e}: \mathcal{A} \rightarrow \mathcal{A}$ to be the identity.

Conversely, given such functors we may define the $\mathcal{T}$-multihoms

$$
\mathbb{A}_{x}\left(a_{1}, \ldots, a_{n} ; b\right)=\mathcal{A}\left(m_{x}\left(a_{1}, \ldots, a_{n}\right), b\right) .
$$

So far this accounts for the objects, the multihoms, the identities, and the substitutions of the form $g\left(f_{1}, \ldots, f_{n}\right)$ and $h(g)$ where $g \in \mathbb{A}_{e}(b ; b)$ for some $b$. This structure satisfies the identity laws as well as associativity of substitution, as far as it is defined. 
Next we turn to the general form of substitution. In our current weakly representable setting, this takes the form of maps

$$
\mathcal{A}\left(m_{x}(\bar{b}), c\right) \times \prod_{i=1}^{n} \mathcal{A}\left(m_{x_{i}}\left(\bar{a}_{i}\right), b_{i}\right) \rightarrow \mathcal{A}\left(m_{x\left(x_{1}, \ldots, x_{n}\right)}\left(\bar{a}_{1}, \ldots, \bar{a}_{n}\right), c\right)
$$

natural in all variables. By naturality in $\left(b_{1}, \ldots, b_{n}\right)$ and the Yoneda lemma that amounts to giving natural maps

$$
\mathcal{A}\left(m_{x}\left(m_{x_{1}}\left(\bar{a}_{1}\right), \ldots, m_{x_{n}}\left(\bar{a}_{n}\right)\right), c\right) \rightarrow \mathcal{A}\left(m_{x\left(x_{1}, \ldots, x_{n}\right)}\left(\bar{a}_{1}, \ldots, \bar{a}_{n}\right), c\right)
$$

and now by naturality in $c$ and the Yoneda lemma this amounts to giving natural maps

$$
m_{x\left(x_{1}, \ldots, x_{n}\right)}\left(\bar{a}_{1}, \ldots, \bar{a}_{n}\right) \rightarrow m_{x}\left(m_{x_{1}}\left(\bar{a}_{1}\right), \ldots, m_{x_{n}}\left(\bar{a}_{n}\right)\right)
$$

or, in other words, to a natural transformation $\tilde{m}$ as in (5.1).

The coassociativity condition for $\tilde{m}$ is equivalent to associativity of substitution.

This defines a bijective correspondence between normal colax $\mathcal{T}^{*}$-algebras and weakly representable $\mathcal{T}$-multicategories, with chosen representations, including the canonical choice of representatives for the $\mathbb{A}_{e}(a ;-)$.

Now suppose that $\mathbb{A}$ and $\mathbb{B}$ are weakly representable $\mathcal{T}$-multicategories corresponding to normal colax $\mathcal{T}^{*}$-algebras $\mathcal{A}$ and $\mathcal{B}$.

What is needed to give a morphism $\mathbb{A} \rightarrow \mathbb{B}$ of $\mathcal{T}$-multicategories? First of all there is an assignment $a \mapsto F a$ on objects. Next there are maps $F_{x}: \mathbb{A}_{x}\left(a_{1}, \ldots, a_{n} ; b\right) \rightarrow \mathbb{B}_{x}\left(F a_{1}, \ldots, F a_{n} ; F b\right)$; in particular, there are maps $\mathbb{A}_{e}(a ; b) \rightarrow \mathbb{B}_{e}(F a ; F b)$ defining a functor $F: \mathcal{A} \rightarrow \mathcal{B}$. The remaining $F_{x}$ correspond, via Yoneda, to maps

$$
m_{x}\left(F a_{1}, \ldots, F a_{n}\right) \stackrel{\tilde{F}}{\longrightarrow} F m_{x}\left(a_{1}, \ldots, a_{n}\right) .
$$

The functoriality condition on the $F_{x}$ corresponds to the associativity condition for the $\tilde{F}$ to define a morphism of normal colax $\mathcal{T}^{*}$-algebras.

The case of 2-cells follows similarly using Yoneda once again.

5.1. The skew case. By Theorem 5.5 we know that the 2-category of normal colax $\mathcal{R}^{*}$-algebras is equivalent to the 2 -category of weakly representable $\mathcal{R}$-multicategories. From now on, we shall be more interested in $\mathcal{R}^{*}$ than $\mathcal{R}$; it is therefore convenient to rename it $\mathcal{L}$. In particular $\mathcal{L}_{0}=\left\{l_{0}\right\}$ and $\mathcal{L}_{n}=\left\{\lambda_{n}: l_{n} \rightarrow t_{n}\right\}$ for $n>0$.

The following is the colax $\mathcal{L}$-algebra version of left representability; it appeared in [5].

Definition 5.6. An LBC-algebra is a normal colax $\mathcal{L}$-algebra for which the maps $\Gamma_{t, 1, x_{n}}: m_{x}\left(a_{1}, \ldots, a_{n+1}\right) \rightarrow m_{t}\left(m_{x}\left(a_{1}, \ldots, a_{n}\right), a_{n+1}\right)$ are identities for all $n$ and all $x \in \mathcal{L}_{n}$.

In the name LBC-algebra, the "LB" stands for left-bracketed and the "C" for colax; see [5]. We define LBC-Alg to be the full sub-2-category of nColax-L-Alg $\mathbf{l g}_{\ell}$ consisting of the LBC-algebras.

Theorem 5.7. The 2-equivalence $\mathbf{n C o l a x}-\mathbf{L}-\mathbf{A} \lg _{\ell} \simeq \mathbf{R}$-Mult restricts to a 2-equivalence between $\mathbf{L B C}$-Alg and the full sub-2-category of $\mathbf{R}$-Mult consisting of the left representable skew multicategories. 
Proof. Given an LBC-algebra $\mathcal{A}$, the corresponding weakly representable $\mathcal{R}$-multicategory $\mathbb{A}$ is related to it as in the following equation.

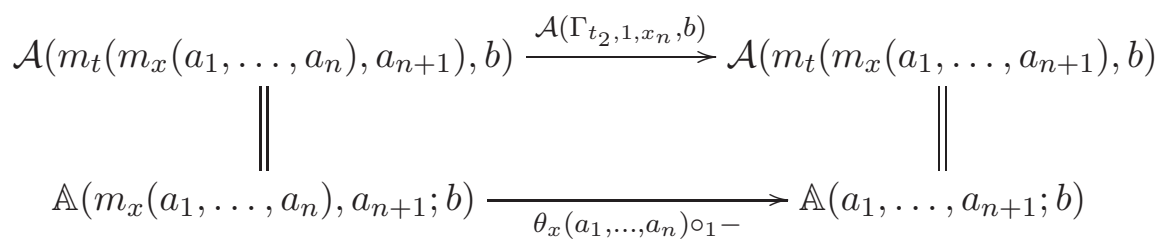

Therefore by Proposition 4.5 (4) $\mathbb{A}$ is left representable. In the other direction, it suffices to show that each left representable skew multicategory arises - up to isomorphism in R-Mult - from an LBC-algebra. By Proposition 4.5 (2) we can equip $\mathbb{A}$ with a choice of multimap classifiers satisfying $m_{t}\left(m_{x}\left(a_{1}, \ldots, a_{n}\right), a_{n+1}\right)=m_{x}\left(a_{1}, \ldots, a_{n+1}\right)$ and with a similar equation for the universal multimaps, and with $m_{t_{1}}(a)=a$. With this choice, the corresponding colax $\mathcal{L}$-algebra satisfies the LBC property, as required.

\section{Skew MUlticAtegories Versus SKeW MONOIDAL CATEGORIES AND SKEW CLOSED CATEGORIES}

We are now in a position to prove our first main result, which combines Theorem 5.7 above with Theorem 7.8 of the companion paper [5].

Theorem 6.1. There is a 2-equivalence between the 2-category $\mathbf{S k w}_{\ell}$ and the full sub 2-category of $\mathbf{R}$-Mult consisting of the left representable skew multicategories.

Proof. By [5, Theorem 7.8] we have a 2-equivalence Skew $_{\ell} \simeq$ LBC-Alg. By Theorem 6.1 above we have a 2-equivalence between LBC-Alg and the full sub-2-category of R-Mult consisting of the left representable skew multicategories. Combining these gives the result.

We now break down the above processes to give a direct description of the relationship between skew monoidal categories and left representable skew multicategories.

6.1. From a skew monoidal category to a left representable skew multicategory. Let $\mathcal{C}$ be a skew monoidal category with unit $i$. We write $a_{1} \ldots a_{n}$ for the left bracketed tensor product in $\mathcal{C}$; thus $a_{1} a_{2}$ denotes the usual tensor product with the formula $a_{1} \ldots a_{n} a_{n+1}=\left(a_{1} \ldots a_{n}\right) a_{n+1}$ determining the higher bracketings.

The corresponding LBC-algebra structure on $\mathcal{C}$ has

$$
\begin{aligned}
m_{\ell}(-) & =i \\
m_{t}\left(a_{1}, \ldots, a_{n}\right) & =a_{1} \ldots a_{n} \\
m_{\ell}\left(a_{1}, \ldots, a_{n}\right) & =i a_{1} \ldots a_{n}
\end{aligned}
$$

with $m_{\lambda}\left(a_{1}, \ldots, a_{n}\right): m_{\ell}\left(a_{1}, \ldots, a_{n}\right) \rightarrow m_{t}\left(a_{1}, \ldots, a_{n}\right)$ given by

$$
i a_{1} \ldots a_{n} \stackrel{\lambda_{a_{1}} a_{2} \ldots a_{n}}{\longrightarrow} a_{1} \ldots a_{n}
$$


The substitution morphisms

$$
m_{x\left(x_{1}, \ldots, x_{n}\right)}\left(\bar{a}_{1}, \ldots, \bar{a}_{n}\right) \stackrel{\Gamma_{x, x_{1}, \ldots, x_{n}}}{\longrightarrow} m_{x}\left(m_{x_{1}}\left(\bar{a}_{1}\right), \ldots, m_{x_{n}}\left(\bar{a}_{n}\right)\right)
$$

are the unique natural families definable for each skew monoidal $\mathcal{C}$ naturally in $\mathcal{C}$. These are obtained by repeated applications of the right unit maps $\rho$ followed by applications of associativity maps $\alpha$, each possibly tensored on either side. For instance $m_{\ell}(a, b, c, d) \rightarrow m_{\ell}\left(m_{t}(a, b), m_{\ell}(c, d)\right)$ is the map given by

$$
(((i a) b) c) d \stackrel{((i(a b)) \rho) d}{\longrightarrow}(((i a) b)(i c)) d \stackrel{((\alpha(i c)) d}{\longrightarrow}(((i(a b))(i c)) d \stackrel{\alpha}{\longrightarrow}(i(a b))((i c) d) .
$$

See Section 7.3 of the companion paper [5] for further details on the LBCalgebra associated to a skew monoidal category.

Accordingly, the corresponding skew multicategory $\mathbb{C}$ has

$$
\begin{aligned}
\mathbb{C}(; a) & =\mathcal{C}(i, a) \\
\mathbb{C}_{t}\left(a_{1}, \ldots, a_{n} ; b\right) & =\mathcal{C}\left(a_{1} \ldots a_{n}, b\right) \\
\mathbb{C}_{\ell}\left(a_{1}, \ldots, a_{n} ; b\right) & =\mathcal{C}\left(i a_{1} \ldots a_{n}, b\right)
\end{aligned}
$$

with $j_{\bar{a}, b}: \mathbb{C}_{t}\left(a_{1}, \ldots, a_{n} ; b\right) \rightarrow \mathbb{C}_{\ell}\left(a_{1}, \ldots, a_{n} ; b\right)$ given by

$$
\mathcal{C}\left(a_{1} \ldots a_{n}, b\right) \stackrel{-\circ \lambda_{a_{1}} a_{2} \ldots a_{n}}{\longrightarrow} \mathcal{C}\left(i a_{1} \ldots a_{n}, b\right) .
$$

The substitution morphisms send

$$
\left(f, g_{1}, \ldots, g_{n}\right) \in \mathcal{C}\left(m_{x}(\bar{b}), c\right) \times \prod_{i=1}^{n} \mathcal{C}\left(m_{x_{i}}\left(\bar{a}_{i}\right), b_{i}\right)
$$

to the composite $f \circ m_{x}\left(g_{1}, \ldots, g_{n}\right) \circ \Gamma_{x, x_{1}, \ldots, x_{n}}\left(\bar{a}_{1}, \ldots, \bar{a}_{n}\right)$.

6.2. From a left representable skew multicategory to a skew monoidal category. This construction is more straightforward and we give it directly, without mentioning the intermediate colax $\mathcal{L}$-algebra structure explicitly. Let $\mathcal{C}$ be a left representable skew multicategory with $\mathcal{C}$ its underlying category.

Tight binary multimap classifiers $a \otimes b$ give representations

$$
\mathcal{C}(a \otimes b, c) \cong \mathbb{C}_{t}(a, b ; c)
$$

and we write $\theta_{t}(a, b) \in \mathbb{C}_{t}(a, b ; a \otimes b)$ for the universal multimap. Functoriality of the right hand side transports to yield a functor $\otimes: \mathcal{C}^{2} \rightarrow \mathcal{C}$.

The nullary map classifier $i$ is defined by a representation

$$
\mathcal{C}(i, a) \cong \mathbb{C}_{\ell}(-; a)
$$

with universal multimap $\theta_{\ell_{0}} \in \mathbb{C}_{\ell}(-; i)$. The object $i$ plays the role of the unit.

- By left representability we have

$$
\mathcal{C}((a \otimes b) \otimes c, d) \cong \mathbb{C}_{t}(a \otimes b, c ; d) \cong \mathbb{C}_{t}(a, b, c ; d) .
$$

Taking $d=a \otimes(b \otimes c)$ and

$$
\theta_{t}(a, b \otimes c) \circ_{2} \theta_{t}(b, c) \in \mathbb{C}_{t}(a, b, c ; a \otimes(b \otimes c))
$$

then gives rise to the associator $\alpha:(a \otimes b) \otimes c \rightarrow a \otimes(b \otimes c)$. 
- By left representability we have

$$
\mathcal{C}(i \otimes a, b) \cong \mathbb{C}_{t}(i, a ; b) \cong \mathbb{C}_{\ell}(a ; b)
$$

and now taking $b=a$ and the image of the identity $1_{a}$ under $\mathbb{C}_{t}(a ; a) \rightarrow \mathbb{C}_{\ell}(a ; a)$ yields the left unit map $\lambda: i \otimes a \rightarrow a$.

- The right unit map $\rho: a \rightarrow a \otimes i$ is the composite $\theta_{t}(a, i) \circ_{2} \theta_{\ell_{0}} \in$ $\mathbb{C}_{t}(a ; a \otimes i)$.

The left unit map admits another interpretation worth mentioning and which follows immediately from its construction above.

Proposition 6.2. Let $\mathbb{C}$ be a left representable skew multicategory. The identity on objects functor $j: \mathcal{C} \rightarrow \mathcal{C}_{\ell}$ has a left adjoint, whose counit is the left unit map $\lambda: i \otimes a \rightarrow a$ for the corresponding skew monoidal structure.

Accordingly $i \otimes a$ classifies loose unary maps. In particular, in the setting of a pseudo-commutative 2-monad $T$, wherein the identity on objects functor is the inclusion $j: \mathbf{T}-\mathbf{A} \lg _{s} \rightarrow \mathbf{T}$-Alg viewing strict morphisms as pseudomorphisms, the tensor product $i \otimes a$ is the pseudomorphism classifier 3.

We can specialise the equivalence of Theorem6.1 as follows. We can identify multicategories with skew multicategories all of whose multimorphisms are tight; thus we can speak of a left representable multicategory. Recall that a skew monoidal category $\mathcal{C}$ is said to be left normal if the left unit map $\lambda: i \otimes a \rightarrow a$ is invertible.

Theorem 6.3. There is a 2-equivalence between the 2-categories of left normal skew monoidal categories and of left representable multicategories.

Proof. The 2-functor $\iota$ : Mult $\rightarrow$ R-Mult viewing multicategories $\mathbb{C}$ as skew multicategories in which all multimaps are tight exhibits Mult as a full sub2 -category of R-Mult. In such a $\mathbb{C}$ the inclusion $j: \mathcal{C} \rightarrow \mathcal{C}_{\ell}$ is the identity whereby its left adjoint has invertible counit. Since in the left representable case Proposition 6.2 ensures that the counit is $\lambda: i \otimes a \rightarrow a$, we conclude that the associated skew monoidal category is left normal.

In the opposite direction let $\mathcal{C}$ be left normal skew monoidal. By their construction in Section 6.1 the components $j_{\bar{a}, b}$ are the maps

$$
\mathcal{C}\left(a_{1} \ldots a_{n}, b\right) \stackrel{-\circ \lambda_{a_{1}} a_{2} \ldots a_{n}}{\longrightarrow} \mathcal{C}\left(i a_{1} \ldots a_{n}, b\right) .
$$

and therefore are invertible whenever $\mathcal{C}$ is left normal. Now the forgetful $U:$ R-Mult $\rightarrow$ Mult has right adjoint $\iota$ and the unit component of the adjunction is invertible precisely at those $\mathcal{C}$ with the above property; thus $\mathcal{C}$ is isomorphic to such a multicategory. It follows that the 2-equivalence of Theorem 6.1 restricts to yield the desired one.

Thus the skew aspect of a skew multicategory arises from the (possible) failure of left normality in the corresponding skew monoidal category.

6.3. Skew monoidal closed categories. Recall [26] that a skew monoidal category $\mathcal{C}$ is said to be closed if for all $b, c \in \mathcal{C}$ there exist an object $[b, c]$ and morphism $e_{b, c}:[b, c] \otimes b \rightarrow c$ such that the induced function 


$$
e_{b, c} \circ\left(-\otimes 1_{b}\right): \mathcal{C}(a,[b, c]) \rightarrow \mathcal{C}(a \otimes b, c)
$$

is a bijection for all $a$.

Theorem 6.4. The 2-equivalence of Theorem 6.1 restricts to a 2-equivalence between the 2-category $\mathbf{S k e w \mathbf { M C l }} \mathbf{l}_{\ell}$ of closed skew monoidal categories and the full sub-2-category of $\mathbf{R}$-Mult consisting of the left representable closed skew multicategories.

Proof. We must show that a left representable skew multicategory $\mathbb{C}$ is closed if and only if the corresponding skew monoidal category $\mathcal{C}$ is so. A tight multimap $e_{b, c} \in \mathbb{C}_{t}([b, c], b ; c)$ is precisely a morphism $e_{b, c}:[b, c] \otimes b \rightarrow c \in \mathcal{C}$. Expressed in terms of $\mathbb{C}$ the family $\left\{e_{b, c} ; b, c \in \mathbb{C}\right\}$ exhibits the skew monoidal $\mathcal{C}$ as closed just when the induced function

$$
e_{b, c} \circ_{1}-: \mathbb{C}_{t}(a,[b, c]) \rightarrow \mathbb{C}_{t}(a, b ; c)
$$

is a bijection for all $a$. Now this is certainly required for $\mathbb{C}$ to be a closed skew multicategory but the full condition asks that the bottom row below

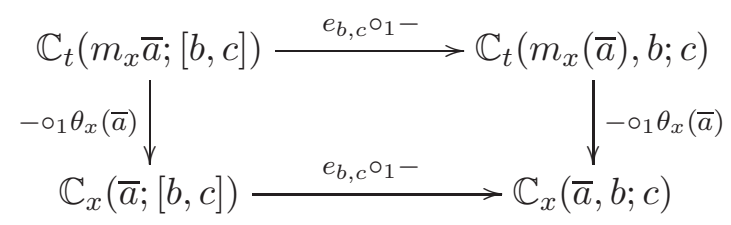

is a bijection for all $x, \bar{a}$. By left representability, however, the universal multimap $\theta_{x}(\bar{a}) \in \mathbb{C}_{x}\left(\bar{a} ; m_{x}(\bar{a})\right)$ induces bijections in the columns; since the diagram commutes the top row is a bijection just when the bottom is one, thus $\mathbb{C}$ is closed just when $\mathcal{C}$ is so.

A natural class of skew multicategories consists of those for which each

$$
j_{\bar{a}, b}: \mathbb{C}_{t}(\bar{a}, b) \rightarrow \mathbb{C}_{\ell}(\bar{a}, b)
$$

is an inclusion - for, as noted in Proposition 4.3, these are just multicategories equipped with a subcollection of tight morphisms closed under substitution in the first variable. By an argument similar to Theorem 6.3, the left representable amongst these correspond to skew monoidal categories for which

$$
\lambda_{a_{1}} a_{2} \ldots a_{n}: i a_{1} \ldots a_{n} \rightarrow a_{1} \ldots a_{n}
$$

is an epimorphism for all non-empty tuples $\bar{a}$, wherein the above morphism involves left bracketings. In the closed skew monoidal case this simplifies since each $-\otimes a_{i}$ preserves epimorphisms. We record the result in that setting, which refines Theorem 6.4 .

Theorem 6.5. There is a 2-equivalence between closed skew monoidal categories whose left unit maps $\lambda: i \otimes a \rightarrow a$ are epimorphisms and those skew multicategories $\mathbb{C}$ with each $j_{\bar{a}, b}: \mathbb{C}_{t}(\bar{a}, b) \rightarrow \mathbb{C}_{\ell}(\bar{a}, b)$ is an inclusion. 
6.4. Skew closed categories versus closed skew multicategories with unit. A skew closed category [26] consists of a category $\mathcal{C}$ equipped with a functor $[-,-]: \mathcal{C}^{o p} \times \mathcal{C} \rightarrow \mathcal{C}$ and object $i$ together with natural transformations

$$
\begin{aligned}
& {[b, c] \stackrel{L}{\longrightarrow}[[a, b],[a, c]]} \\
& {[i, a] \stackrel{I}{\longrightarrow} a} \\
& i \stackrel{J}{\longrightarrow}[a, a]
\end{aligned}
$$

subject to five axioms [26]. If the components $I:[i, a] \rightarrow a$ are invertible as well as the functions $\mathcal{C}(J, 1) \circ[a,-]: \mathcal{C}(a, b) \rightarrow \mathcal{C}(i,[a, b])$, the skew closed category is said to be closed 1

Between skew closed categories are closed functors, which involve morphisms $[F a, F b] \rightarrow F[a, b]$ and $i \rightarrow F i$, and closed transformations. All together, these form a 2-category $\mathbf{S k e w}_{\ell} \mathbf{C l}_{\ell}$.

In Theorem 5.1 of of [24], Manzyuk established a correspondence between closed categories and closed multicategories with unit. The following theorem, which builds on work of [4, gives the skew version of Manzyuk's theorem. Our argument, which is rather different in character to Manzyuk's, essentially treats the skew closed case as a special case of the skew monoidal case and can easily be adapted to give an alternative proof of his result.

Theorem 6.6. There is a 2-equivalence between the 2-category $\mathbf{S k e w} \mathbf{C l}_{\ell}$ and the full sub 2-category of R-Mult consisting of the closed skew multicategories with unit.

Proof. We give only the core details of the proof. Our argument will proceed in three steps:

Step (I) skew closed structures on $\mathcal{C}$ correspond to certain right skew monoidal structures on $[\mathcal{C}$, Set $]$;

Step (II) the right skew monoidal structures of (I) correspond to certain lax $\mathcal{R}$-algebra structures;

Step (III) the lax $\mathcal{R}$-algebra structures of (II) correspond to closed skew multicategories with unit, whose underlying category is $\mathcal{C}$.

Step (I) is due to Street [26] and builds on work of Day [8]. A (left) skew promonoidal structure on $\mathcal{C}$ is a left skew pseudomonoid in the monoidal bicategory of profunctors: such involves structure functors $P: \mathcal{C}^{o p} \times \mathcal{C}^{o p} \times$ $\mathcal{C} \rightarrow$ Set and $J: \mathcal{C} \rightarrow$ Set plus three coherence constraints satisfying five equations [26]. By Proposition 22 of [26] each skew closed structure on $\mathcal{C}$ determines a skew promonoidal structure with $P(a, b, c)=\mathcal{C}(a,[b, c])$ and $J a=\mathcal{C}(i, a)$. By the same proposition skew closed structures on $\mathcal{C}$ can be identified with promonoidal structures for which each $P(-, b, c): \mathcal{C}^{o p} \rightarrow$ Set and $J: \mathcal{C} \rightarrow$ Set are representable. Now left skew promonoidal structures on $\mathcal{C}$ correspond to right skew monoidal structures on $[\mathcal{C}$, Set $]$ whose tensor product is cocontinuous in each variable: the right skew monoidal structure on $[\mathcal{C}$, Set $]$ has a convolution tensor product given by the left Kan extension

\footnotetext{
${ }^{1}$ The original definition of closed category [10] involved an underlying functor to Set. Above we refer to the modified definition of [21].
} 
$m=\operatorname{lan}_{y^{2}} P$

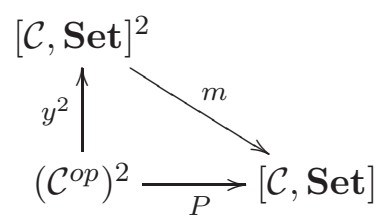

and unit $J$. Putting this together we conclude that skew closed structures on $\mathcal{C}$ amount to

(1) Right skew monoidal structures $([\mathcal{C}$, Set $], m, J)$ such that $m$ is cocontinuous in each variable, such that $m(y-, y b)(c): \mathcal{C}^{o p} \rightarrow$ Set is representable, and such that $J: \mathcal{C} \rightarrow$ Set is representable.

By Theorem 7.8 of the companion paper [5], left skew monoidal structures on a category correspond to LBC-algebras - normal colax $\mathcal{L}$-algebras whose substitution maps

$$
m_{x_{n+1}}(\bar{a}, b) \stackrel{\Gamma_{t_{2}, 1, x_{n}}}{\longrightarrow} m_{t_{2}}\left(m_{x_{n}}(\bar{a}), b\right)
$$

are identities. If these are merely isomorphisms rather than identities then, by an easy transport of structure argument, we can produce an isomorphic normal colax structure on $\mathcal{C}$ satisfying the stricter condition. Accordingly, left skew monoidal structures equally correspond to normal colax $\mathcal{L}$-algebras with (6.1) invertible. The dual result is that right skew monoidal structures on $[\mathcal{C}$, Set $]$ correspond to normal lax $\mathcal{R}$-algebra structures on $[\mathcal{C}$, Set $]$ for which the substitution maps

$$
m_{t_{2}}\left(m_{x_{n}}(\bar{a}), b\right) \stackrel{\Gamma_{t_{2}, 1, x_{n}}}{\longrightarrow} m_{x_{n+1}}(\bar{a}, b)
$$

are invertible 2 The lax structure associated to $([\mathcal{C}$, Set $], m, J)$ has $m_{t_{2}}=$ $m$ and $m_{l_{0}}=J$. The natural isomorphisms $m_{x_{n+1}}(\bar{a}, b) \cong m_{t_{2}}\left(m_{x_{n}}(\bar{a}), b\right)$ inductively ensure that each $m_{x}:[\mathcal{C} \text {, Set }]^{n} \rightarrow[\mathcal{C}$, Set $]$ is cocontinuous in each variable if $m$ is. Accordingly, right skew monoidal structures as per (1) correspond to

(2) Normal lax $\mathcal{R}$-algebra structures on $[\mathcal{C}$, Set $]$ with $m_{x}$ cocontinuous in each variable for all $x$ and such that

(a) The functors $m_{t_{2}}(y-, y b)(c): \mathcal{C}^{o p} \rightarrow$ Set and $m_{l_{0}}: \mathcal{C} \rightarrow$ Set are representable.

(b) The substitution maps (6.2) are invertible.

Accordingly, it remains to establish a correspondence between closed skew multicategories with unit on $\mathcal{C}$ and structures as in (2) above. In fact, the core of this correspondence holds for a general Cat-operad $\mathcal{T}$ : $\mathcal{T}$-multicategory structures on $\mathcal{C}$ correspond to normal lax $\mathcal{T}$-algebra structures on $[\mathcal{C}$, Set $]$ for which $m_{x}:[\mathcal{C} \text {, Set }]^{n} \rightarrow[\mathcal{C}$, Set $]$ is cocontinuous in each variable for each $x \in \mathcal{T}_{n}$.

To see this, first recall from Proposition 3.4 that for a $\mathcal{T}$-multicategory $\mathbb{C}$, the collections of multimaps extend uniquely to functors

$$
\mathbb{C}_{-}(-;-): \mathcal{T}_{n} \times\left(\mathcal{C}^{n}\right)^{o p} \times \mathcal{C} \rightarrow \text { Set. }
$$

\footnotetext{
${ }^{2} \mathrm{~A}$ lax $\mathcal{R}$-algebra corresponds to a colax $\mathcal{L}$-algebra structure on the opposite category - accordingly its substitution maps point in the opposite direction.
} 
such that $\mathbb{C}_{e}(-;-)=\mathcal{C}(-;-)$ and with respect to which substitution becomes natural in each variable. This allows us to identify $\mathcal{T}$-multicategory structures on $\mathcal{C}$ with multicategories equipped with such extensions. Given such a $\mathbb{C}$ the convolution lax $\mathcal{T}$-algebra structure on $[\mathcal{C}$, Set $]$ has $m_{x}$ given by the left Kan extension

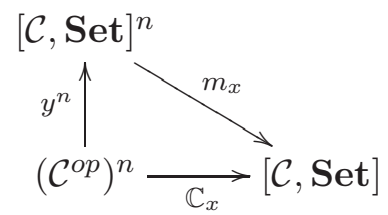

Accordingly $m_{x}$ is cocontinuous in each variable and is given by

$$
m_{x}\left(A_{1}, \ldots, A_{n}\right)=\int^{a_{1}, \ldots, a_{n} \in \mathcal{C}} A_{1} a_{1} \times \ldots A_{n} a_{n} \times \mathbb{C}_{x}\left(a_{1}, \ldots, a_{n} ;-\right) .
$$

We sometimes abbreviate this by $\int^{\bar{a}} \bar{A} \bar{a} \times \mathbb{C}_{x}(\bar{a} ;-)$. Since $y^{n}$ is fully faithful we have $m_{x}\left(y a_{1}, \ldots, y a_{n}\right) \cong \mathbb{C}_{x}\left(a_{1}, \ldots, a_{n} ;-\right)$.

Now $\mathbb{C}_{e}=y: \mathcal{C}^{o p} \rightarrow[\mathcal{C}$, Set $]$; hence we may, and do, set $m_{e}=1$ so as to obtain normality. The components $m_{f}: m_{x} \rightarrow m_{y}$ for $f: x \rightarrow y \in \mathcal{T}_{n}$ satisfy the obvious formula. The component of substitution $m_{x}\left(m_{x 1}, \ldots, m_{x n}\right) \rightarrow$ $m_{x\left(x_{1}, \ldots, x_{n}\right)}$ at $\left(\overline{A_{1}}, \ldots, \overline{A_{n}}\right)$ is the composite

$$
\begin{aligned}
& \int^{\bar{b}}\left(\int^{\overline{a_{1}}} \overline{A_{1}} \overline{a_{1}} \times \mathbb{C}_{x_{1}}\left(\overline{a_{1}} ; b_{1}\right) \times \ldots \times \int^{\overline{a_{n}}} \overline{A_{n}} \overline{a_{n}} \times \mathbb{C}_{x_{1}}\left(\overline{a_{n}} ; b_{n}\right)\right) \times \mathbb{C}_{x}(\bar{b} ;-)
\end{aligned}
$$

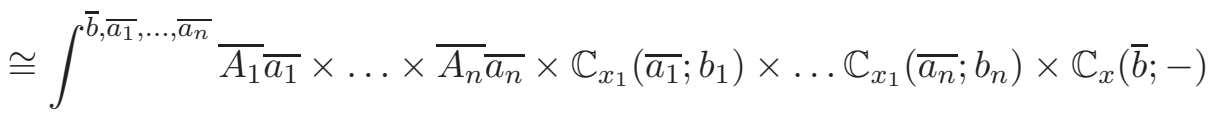

$$
\begin{aligned}
& \longrightarrow \int^{\overline{a_{1}}, \ldots, \overline{a_{n}}} \overline{A_{1}} \overline{a_{1}} \times \ldots \times \overline{A_{n}} \overline{a_{n}} \times \mathbb{C}_{x\left(x_{1}, \ldots, x_{n}\right)}\left(\overline{a_{1}}, \ldots, \overline{a_{n}} ;-\right)
\end{aligned}
$$

whose second component is a coend of substitution maps. On representables it returns, up to natural isomorphism, the substitution maps for $\mathbb{C}$. The lax $\mathcal{T}$-algebra axioms are easily verified: since they assert the equality of composite natural transformations between functors cocontinuous in each variable it is enough to check they hold at representables, where they amount to the axioms for a $\mathcal{T}$-multicategory. We omit the straightforward converse construction, which is obtained by restriction along powers of the Yoneda embedding.

Finally we specialise to $\mathcal{T}=\mathcal{R}$. By the above analysis we have a correspondence between $\mathcal{R}$-multicategory structure on $\mathcal{C}$ and normal lax $\mathcal{R}$ algebra structure on $[\mathcal{C}$, Set $]$ for which each $m_{x}$ is cocontinuous in each variable. It remains, then, to prove that it restricts to a correspondence between closed skew multicategories with unit and lax $\mathcal{R}$-algebras having the properties $(2 \mathrm{a})$ and $(2 \mathrm{~b})$.

Let $\mathbb{C}$ be an $\mathcal{R}$-multicategory. We investigate what the conditions (2a) and $(2 \mathrm{~b})$, interpreted at the associated lax $\mathcal{R}$-algebra, mean for $\mathbb{C}$ itself. The representability conditions of (2a) simply amount to the existence of a nullary map classifier and objects $[b, c]$ equipped with isomorphisms

$$
\mathbb{C}_{e}(a,[b, c]) \cong \mathbb{C}_{t_{2}}(a, b ; c)
$$


natural in $a$. Letting $e_{b, c} \in \mathbb{C}_{t_{2}}([b, c], b)$ denote the unit of the representation, we must show that the maps

$$
\mathbb{C}_{x}(\bar{a},[b, c]) \stackrel{e_{b, c} \circ_{1}-}{\longrightarrow} \mathbb{C}_{x_{n+1}}(\bar{a}, b ; c)
$$

are invertible - that is, $\mathbb{C}$ is closed - if and only if the associated lax $\mathcal{T}$ algebra satisfies $(2 b)$.

By cocontinuity of $m_{x}$ in each variable, the substitution maps of (2b) will be invertible in all components just when they are so at representables; that is, just when the map

$$
m_{t_{2}}\left(m_{x_{n}}(y \bar{a}), y b\right) \stackrel{\Gamma_{t_{2}, 1, x_{n}}}{\longrightarrow} m_{x_{n+1}}(y \bar{a}, y b)
$$

is so for all $\bar{a} \in \mathcal{C}^{n}$ and $b \in \mathcal{C}$. This map is induced by multicategorical substitution: at $c \in \mathcal{C}$ it has component

$$
\int^{d, f} \mathbb{C}_{x}(\bar{a} ; d) \times \mathbb{C}_{e}(b ; f) \times \mathbb{C}_{t_{2}}(d, f ; c) \rightarrow \mathbb{C}_{x_{n+1}}(\bar{a}, b ; c)
$$

which, applying Yoneda to the domain, is isomorphic to

$$
\circ_{1}: \int^{d} \mathbb{C}_{x}(\bar{a} ; d) \times \mathbb{C}_{t_{2}}(d, b ; c) \rightarrow \mathbb{C}_{x_{n+1}}(\bar{a}, b ; c)
$$

Accordingly we must show that these last maps are invertible just when those in (6.5) are. Now in the commutative diagram below the left vertical map is a Yoneda isomorphism whilst invertibility of the top horizontal map follows from (6.4).

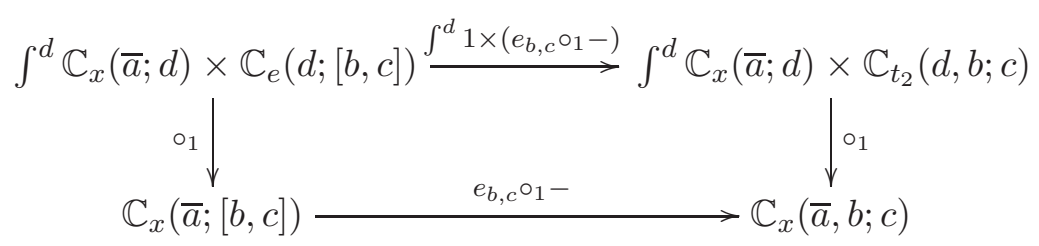

Therefore the right vertical map is invertible if and only if the bottom horizontal map is so.

\section{REFERENCES}

[1] Aguiar, M., Haim, M., and López-Franco, I. Monads on higher monoidal categories. Applied Categorical Structures, https://doi.org/10.1007/s10485-017-9497-8, 2017.

[2] Michael Batanin and Mark Weber. Algebras of higher operads as enriched categories. Appl. Categ. Structures, 19(1):93-135, 2011.

[3] R. Blackwell, G. M. Kelly, and A. J. Power. Two-dimensional monad theory. J. Pure Appl. Algebra, 59(1):1-41, 1989.

[4] John Bourke. Skew structures in 2-category theory and homotopy theory. J. Homotopy Relat. Struct., 12(1):31-81, 2017.

[5] John Bourke and Stephen Lack. Free skew monoidal categories. arXiv:1708.06087, 2017.

[6] John Bourke and Stephen Lack. Symmetries and braidings for skew monoidal categories. In preparation.

[7] G. S. H. Cruttwell and Michael A. Shulman. A unified framework for generalized multicategories. Theory Appl. Categ., 24:No. 21, 580-655, 2010.

[8] Brian Day. Construction of Biclosed Categories. PhD Thesis, University of New South Wales, 1970. 
[9] Brian Day and Ross Street. Lax monoids, pseudo-operads, and convolution. In $D i$ agrammatic morphisms and applications (San Francisco, CA, 2000), volume 318 of Contemp. Math., pages 75-96. Amer. Math. Soc., Providence, RI, 2003.

[10] Samuel Eilenberg and G. Max Kelly. Closed categories. In Proc. Conf. Categorical Algebra (La Jolla, Calif., 1965), pages 421-562. Springer, New York, 1966.

[11] A. D. Elmendorf and M. A. Mandell. Rings, modules, and algebras in infinite loop space theory. Adv. Math., 205(1):163-228, 2006.

[12] Claudio Hermida. Representable multicategories. Adv. Math., 151(2):164-225, 2000.

[13] Martin Hyland and John Power. Pseudo-commutative monads and pseudo-closed 2categories. J. Pure Appl. Algebra, 175(1-3):141-185, 2002. Special volume celebrating the 70th birthday of Professor Max Kelly.

[14] G. M. Kelly. On clubs and doctrines. In Category Seminar (Proc. Sem., Sydney, 1972/1973), pages 181-256. Lecture Notes in Math., Vol. 420. Springer, Berlin, 1974.

[15] Stephen Lack and Ross Street. Skew monoidales, skew warpings and quantum categories. Theory Appl. Categ., 26:385-402, 2012.

[16] Stephen Lack and Ross Street. On monads and warpings. Cah. Topol. Géom. Différ. Catég., 55(4):244-266, 2014.

[17] Stephen Lack and Ross Street. A skew-duoidal Eckmann-Hilton argument and quantum categories. Appl. Categ. Structures, 22(5-6):789-803, 2014.

[18] Stephen Lack and Ross Street. Triangulations, orientals, and skew monoidal categories. Adv. Math., 258:351-396, 2014.

[19] Stephen Lack and Ross Street. Skew-monoidal reflection and lifting theorems. Theory Appl. Categ., 30:985-1000, 2015.

[20] Joachim Lambek. Deductive systems and categories. II. Standard constructions and closed categories. In Category Theory, Homology Theory and their Applications, I (Battelle Institute Conference, Seattle, Wash., 1968, Vol. One), pages 76-122. Springer, Berlin, 1969.

[21] Miguel L. Laplaza. Embedding of closed categories into monoidal closed categories. Trans. Amer. Math. Soc., 233:85-91, 1977.

[22] Tom Leinster. Higher operads, higher categories, volume 298 of London Mathematical Society Lecture Note Series. Cambridge University Press, Cambridge, 2004.

[23] Saunders Mac Lane. Natural associativity and commutativity. Rice Univ. Studies, 49(4):28-46, 1963.

[24] Oleksandr Manzyuk. Closed categories vs. closed multicategories. Theory Appl. Categ., 26:No. 5, 132-175, 2012.

[25] Martin Markl. Operads and PROPs. In Handbook of algebra. Vol. 5, volume 5 of Handb. Algebr., pages 87-140. Elsevier/North-Holland, Amsterdam, 2008.

[26] Ross Street. Skew-closed categories. J. Pure Appl. Algebra, 217(6):973-988, 2013.

[27] Kornél Szlachányi. Skew-monoidal categories and bialgebroids. Adv. Math., 231(34):1694-1730, 2012.

Department of Mathematics, Macquarie University NSW 2109, Australia

E-mail address: steve.lack@mq.edu.au 